\title{
Chandra Observation of Abell 2142: Survival of Dense Subcluster Cores in a Merger
}

\section{Citation}

Markevitch, M., T. J. Ponman, P. E. J. Nulsen, M. W. Bautz, D. J. Burke, L. P. David, D. Davis, et al. 2000. "ChandraObservation of Abell 2142: Survival of Dense Subcluster Cores in a Merger." The Astrophysical Journal 541 (2): 542-49. https://doi.org/10.1086/309470.

\section{Permanent link}

http://nrs.harvard.edu/urn-3:HUL.InstRepos:41399845

\section{Terms of Use}

This article was downloaded from Harvard University's DASH repository, and is made available under the terms and conditions applicable to Other Posted Material, as set forth at http:// nrs.harvard.edu/urn-3:HUL.InstRepos:dash.current.terms-of-use\#LAA

\section{Share Your Story}

The Harvard community has made this article openly available.

Please share how this access benefits you. Submit a story.

Accessibility 
The Astrophysical JouRnaL, 541:542-549, 2000 October 1

(C) 2000. The American Astronomical Society. All rights reserved. Printed in U.S.A.

\title{
CHANDRA OBSERVATION OF ABELL 2142: SURVIVAL OF DENSE SUBCLUSTER CORES IN A MERGER
}

\author{
M. Markevitch, ${ }^{1}$ T. J. Ponman, ${ }^{1,2}$ P. E. J. Nulsen,,${ }^{1,3}$ M. W. Bautz, ${ }^{4}$ D. J. Burke, ${ }^{5}$ L. P. David, ${ }^{1}$ D. Davis, ${ }^{4}$ \\ R. H. Donnelly, ${ }^{1}$ W. R. Forman, ${ }^{1}$ C. Jones, ${ }^{1}$ J. KaAstra, ${ }^{6}$ E. KellogG, ${ }^{1}$ D.-W. Kim, ${ }^{1}$ J. KolodZIEJCZAK, $^{7}$ \\ P. Mazzotta, ${ }^{1,8}$ A. Pagliaro, ${ }^{5}$ S. Patel, ${ }^{6}$ L. Van SpeybroecK, ${ }^{1}$ A. Vikhlinin, ${ }^{1,9}{ }^{\text {J. VrtileK, }}{ }^{1}$ \\ M. WISE, ${ }^{4}$ AND P. ZHAO ${ }^{1}$ \\ Received 2000 January 14; accepted 2000 March 27
}

\begin{abstract}
We use Chandra data to map the gas temperature in the central region of the merging cluster A2142. The cluster is markedly nonisothermal; it appears that the central cooling flow has been disturbed but not destroyed by a merger. The X-ray image exhibits two sharp, bow-shaped, shocklike surface brightness edges or gas density discontinuities. However, temperature and pressure profiles across these edges indicate that these are not shock fronts. The pressure is reasonably continuous across these edges, while the entropy jumps in the opposite sense to that in a shock (i.e., the denser side of the edge has lower temperature, and hence lower entropy). Most plausibly, these edges delineate the dense subcluster cores that have survived a merger and ram pressure stripping by the surrounding shock-heated gas.

Subject headings: cooling flows — galaxies: clusters: individual (Abell 2142) — intergalactic medium — $\mathrm{X}$-rays: general
\end{abstract}

\section{INTRODUCTION}

Clusters of galaxies grow through gravitational infall and merger of smaller groups and clusters. During a merger, a significant fraction of the enormous $\left(\sim 10^{63-64}\right.$ ergs) kinetic energy of the colliding subclusters dissipates in the intracluster gas through shock heating, giving rise to strong, but transient, spatial variations of gas temperature and entropy. These variations contain information on the stage, geometry, and velocity of the merger. They also can shed light on physical processes and phenomena occurring in the intracluster medium, including gas bulk flows, destruction of cooling flows, turbulence, and thermal conduction. Given this wealth of information contained in the merger temperature maps, they have in the past few years been a subject of intensive study, both experimental (using ROSAT PSPC and $A S C A$ data, e.g., Henry \& Briel 1996, Markevitch, Sarazin, \& Vikhlinin 1999, and references in those works) and theoretical, using hydrodynamic simulations (e.g., Schindler \& Muller 1993, Roettiger, Burns, \& Stone 1999, and references therein). The measurements reported so far, while revealing, were limited by ROSAT's limited energy coverage and $A S C A$ 's moderate angular resolution. Two new X-ray observatories, Chandra and $X M M$, will overcome these difficulties and provide much more accurate spatially resolved temperature data, adequate for studying the above phenomena.

In this paper, we analyze the first Chandra observation of a merging cluster, $\mathrm{A} 2142(z=0.089)$. This hot $\left(T_{e} \sim 9 \mathrm{keV}\right)$, $\mathrm{X}$-ray-luminous cluster has two bright elliptical galaxies near the center, aligned in the general direction of the X-ray

\footnotetext{
${ }^{1}$ Harvard-Smithsonian Center for Astrophysics, 60 Garden Street, Cambridge, MA 02138.

${ }^{2}$ University of Birmingham, UK.

${ }^{3}$ University of Wollongong, Australia.

${ }^{4}$ MIT.

${ }^{5}$ Institute for Astronomy, University of Hawaii.

${ }^{6}$ SRON, the Netherlands.

${ }^{7}$ NASA/MSFC.

${ }^{8}$ Universita di Roma, Italy.

${ }^{9}$ Space Research Institute, Russian Academy of Science.
}

brightness elongation. The line-of-sight velocities of these galaxies differ by $1840 \mathrm{~km} \mathrm{~s}^{-1}$ (Oegerle, Hill, \& Fitchett 1995), suggesting that the cluster is not in a dynamically relaxed state. The X-ray image of the cluster has a peak indicating a cooling flow. From the ROSAT HRI image, Peres et al. (1998) deduced a cooling flow rate of $72_{-19}^{+14} h^{-2}$ $M_{\odot} \mathrm{yr}^{-1}$. From the ROSAT PSPC image, Buote \& Tsai (1996) argued that this cluster is at a late merger stage. Henry \& Briel (1996) used ROSAT PSPC data to derive a rough gas temperature map for A2142. Since this cluster is too hot for the PSPC to derive accurate temperatures, they adjusted the PSPC gain to make the average temperature equal to that from Ginga and looked for spatial hardness variations. Their temperature map showed azimuthally asymmetric temperature variations, which also is an indication of a merger. A derivation of an $A S C A$ temperature map for this relatively distant cluster was hindered by the presence of a central brightness peak associated with a cooling flow.

Examination of the ROSAT PSPC and HRI images reveals two striking X-ray brightness edges within a few arcminutes northwest and south of the brightness peak, which were not reported in the earlier studies of A2142. The new Chandra data show these intriguing cluster gas features more clearly and allow us to study them in detail, including spectroscopically. Chandra also provides a high-resolution temperature map of the central cluster region. These results are presented below. We use $H_{0}=100 h \mathrm{~km} \mathrm{~s}^{-1} \mathrm{Mpc}^{-1}$ and $q_{0}=0.5$; confidence intervals are one-parameter $90 \%$.

\section{DATA REDUCTION}

A2142 was observed by Chandra during the calibration phase on 1999 August 20 with the ACIS-S detector. ${ }^{10}$ Two similar, consecutive observations (OBSID 1196 and 1228) are combined here. The data were telemetered in Faint mode. Known hot pixels, bad columns, chip node boundaries, and events with $A S C A$ grades 1, 5, and 7 are excluded

\footnotetext{
${ }^{10}$ Chandra Observatory Guide http://asc.harvard.edu/udocs/docs/ docs.html, section "Observatory Guide," "ACIS."
} 

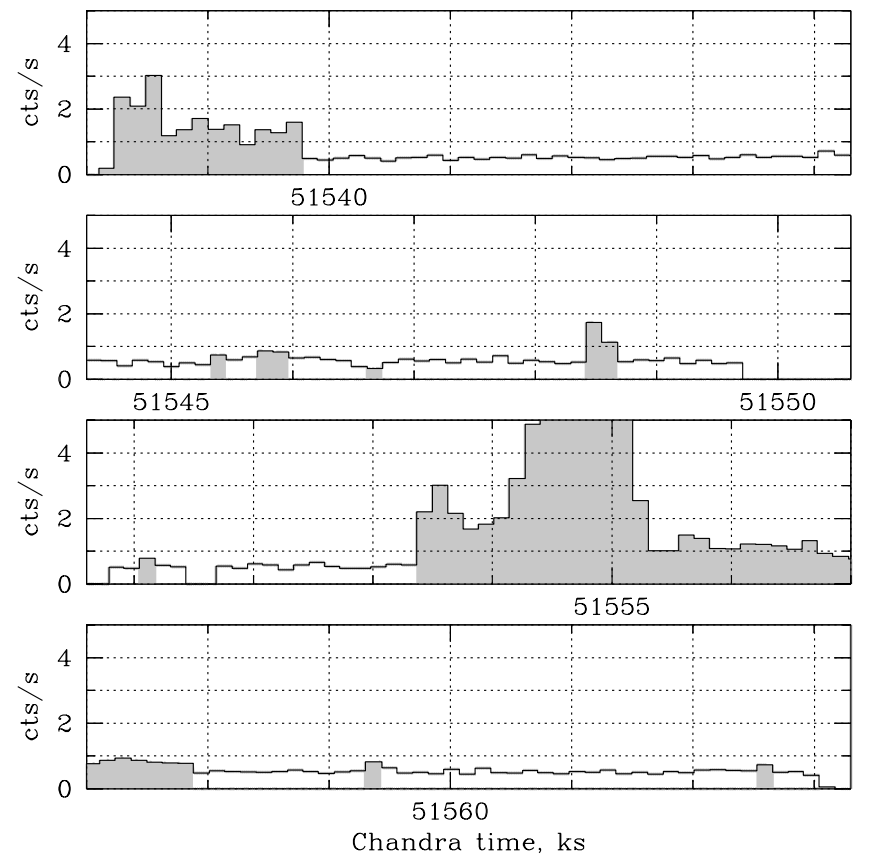

FIG. 1.- Light curve for a region of chip $\mathrm{S} 3$ far from the cluster brightness peak. Bins are $130 \mathrm{~s}$. Shaded intervals of high (or low) background are excluded from the analysis.

from the analysis, along with several short time intervals with incorrect aspect reconstruction. The cluster was centered in the backside-illuminated chip S3 that is susceptible to particle background flares. ${ }^{11}$ For our study of the low surface brightness regions of the cluster, it is critical to exclude any periods with anomalous background. For that, we made a light curve for a region covering $1 / 5$ of the $S 3$ chip far from the cluster peak where the relative background contribution to the flux is largest, using screened events in the $0.3-10 \mathrm{keV}$ energy band (Fig. 1). The light curve shows that most of the time the background is quiescent (approximately half of the flux during these periods is due to the cluster emission in this region of the detector) but there are several flares. We excluded all time intervals when the flux was significantly, by more than $3 \sigma$, above or below the quiescent rate (the flux may be below normal, for example, due to data dropouts). The excluded intervals are shaded in Figure 1. This screening resulted in a total clean exposure of $16.4 \mathrm{ks}$ for the $\mathrm{S} 3 \mathrm{chip}$ (out of a total of $24 \mathrm{ks}$ ). The same flare intervals can be identified from the light curve of another backside-illuminated chip, S1, that also was active during the exposure but has a much smaller cluster contribution. A similar screening of the frontsideilluminated chips, less affected by the flares, resulted in a total clean exposure of $21.3 \mathrm{ks}$ for those chips. In this paper, we limit our imaging analysis to chips $\mathrm{S} 2$ and $\mathrm{S} 3$ and spectral analysis to chip S3.

During the quiescent periods, the particle background is rather constant in time but is nonuniform over the chip (varying by $\sim 30 \%$ on scales of a few arcminutes). To take this nonuniformity into account in our spectral and imaging analysis, we used a background data set composed of several other observations of relatively empty fields with

\footnotetext{
${ }^{11}$ Chandra memo http://asc.harvard.edu/cal/, section "ACIS," “ACIS Background."
}

bright sources removed. Those observations were screened in exactly the same manner as the cluster data. The total exposure of that data set is about $70 \mathrm{ks}$. To be able to extract the background spectra and images in sky coordinates corrected for the observatory dither, chip coordinates of the events from the background data set were converted to the sky coordinate frame of the observation being analyzed. This was done by assigning randomly generated time tags to the background events and applying the corresponding aspect correction. The background spectra or images were then normalized by the ratio of the respective exposures. This procedure yields a background model which is accurate to $\sim 10 \%$ based on comparison to other fields; this uncertainty will be taken into account in our results.

For generating a temperature map ( $\S 3.3)$, we corrected the images for the effect of the source smearing during the periods of CCD frame transfer. While the frame transfer duration $(41 \mathrm{~ms})$ is small compared to the useful exposure $(3.2 \mathrm{~s})$ in each readout cycle, the contamination may be significant for the outer, low surface brightness regions of the cluster that have the same chip $x$ coordinates as the cluster sharp brightness peak. To a first approximation, this effect can be corrected by convolving the ACIS image with the readout trajectory (a line parallel to the chip $y$-axis), multiplying by the ratio of the frame transfer and useful exposures, and subtracting from the uncorrected image. This assumes that the image is not affected by the pileup effect, which is true for most cluster data, including ours.

\section{RESULTS}

\subsection{Image}

An ACIS image of the cluster using the $0.3-10 \mathrm{keV}$ events from chips S2 and S3 is shown in Figure 2 (the cluster peak is in S3). An overlay of the X-ray contours on the DSS optical plate in Figure $2 b$ shows that the cluster brightness peak is slightly offset from the central galaxy (galaxy 201 in the Oegerle et al. notation; we will call it G1) and that the second bright galaxy, hereafter G2 (or galaxy 219 from Oegerle et al.), does not have any comparable gas halo around it. North of G2, there is an X-ray point source coincident with a narrow-tail radio galaxy (Harris, Bahcall, $\&$ Strom 1977).

The image in Figure $2 a$ shows a very regular, elliptical brightness distribution and two striking, elliptical-shaped edges, or abrupt drops, in the surface brightness, one $\sim 3^{\prime}$ northwest of the cluster center and another $\sim 1^{\prime}$ south of the center. We derive gas density and temperature profiles across these interesting structures in $\$ \S 3.4-3.5$.

\subsection{Average Cluster Spectrum}

Before proceeding to the spatially resolved spectroscopy, we fit the overall cluster spectrum to check the consistency with previous studies. For this, we use a spectrum from the entire S3 chip, excluding point sources. This approximately corresponds to an integration radius of $5^{\prime}$. At present, the soft spectral response of the S3 chip is uncertain and we observe significant residual deviations below $E \simeq 0.7 \mathrm{keV}$ for any reasonable spectral models. Therefore, we have chosen to restrict all spectral analysis to energies $1-10 \mathrm{keV}$. The cluster is hot, and this choice does not limit the accuracy of our main results. The spectra were extracted in PI (pulse height-invariant) channels that correct for the gain 


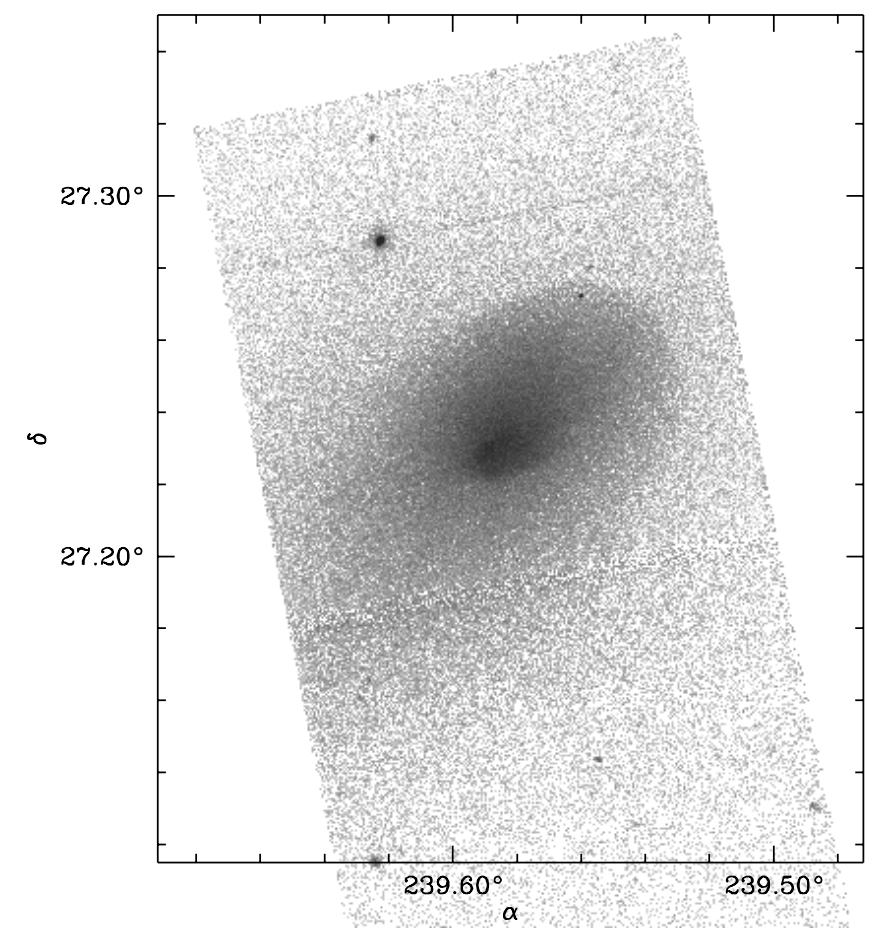

FIG. $2 a$

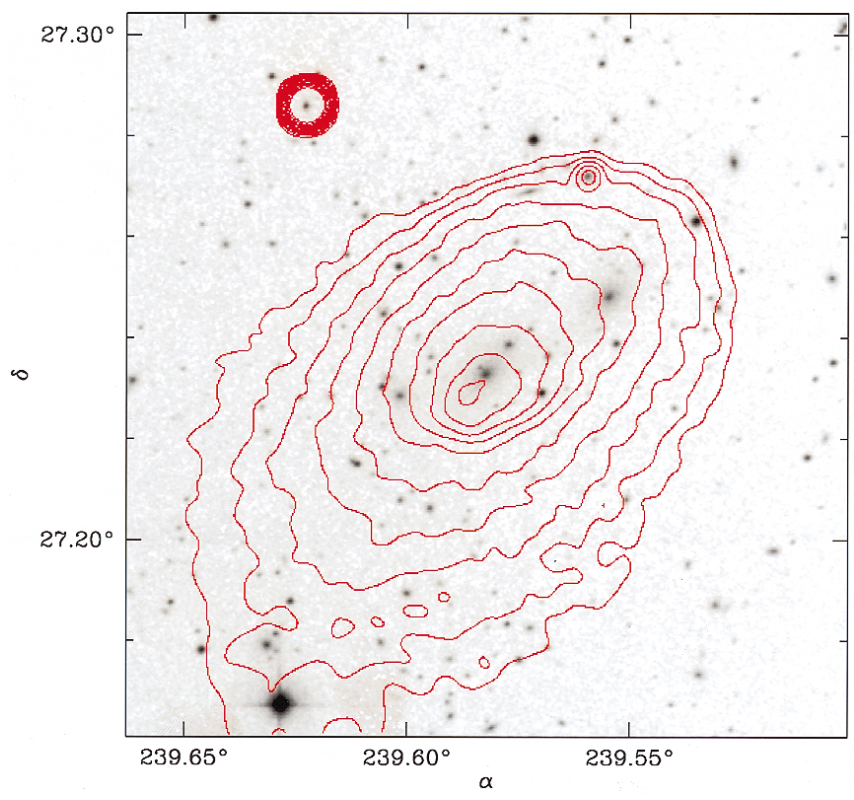

FIG. $2 b$

FIG. 2. - (a) ACIS image of A2142 in the $0.3-10 \mathrm{keV}$ band, binned to 2" pixels and divided by the vignetting map. Only chips S2 and S3 are included. Note the two sharp elliptical brightness edges northwest and south of the cluster peak. A streak of the emission that goes through the bright point source (a Seyfert galaxy - member of the cluster) is the uncorrected track of this source during the CCD frame transfer. (b) A Digitized Sky Survey image with overlaid ACIS X-ray brightness contours (log-spaced by a factor of $\sqrt{2}$ ). The two major galaxies, G1 and G2, are seen at $\alpha=239^{\circ} .5836, \delta=27^{\circ} .2333$ and $\alpha=239^{\circ} .5556$, $\delta=27.2479$, respectively.

difference between the different regions of the CCD. The spectra from both pointings were grouped to have a minimum of 100 counts per bin and fitted simultaneously using the XSPEC package (Arnaud 1996). Model spectra were multiplied by the vignetting factor (auxiliary response) calculated by weighting the position-dependent effective area with the X-ray brightness over the corresponding image region. Fitting results for an absorbed singletemperature thin plasma model (Raymond \& Smith 1977, 1992 revision) and a model with an additional cooling flow component are given in Table 1, where the iron abundance is relative to that of Anders \& Grevesse (1989). Our singletemperature fit is in reasonable agreement with values from Ginga $\left(9.0 \pm 0.3 \mathrm{keV}\right.$ for $N_{\mathrm{H}}=5 \times 10^{20} \mathrm{~cm}^{-2}$; White et al. 1994) and $A S C A\left(8.8 \pm 0.6 \mathrm{keV}\right.$ for $N_{\mathrm{H}}=4.2 \times 10^{20} \mathrm{~cm}^{-2}$; Markevitch et al. 1998). At this stage of the Chandra calibration, and for our qualitative study, the apparent small discrepancy is not a matter of concern; also, the above values correspond to different integration regions for this highly nonisothermal cluster. If we allow for a cooling flow component (see Table 1), our temperature is consistent with a similarly derived $A S C A$ value, $9.3_{-0.7}^{+1.3} \mathrm{keV}$ (Allen \& Fabian 1998), and the cooling rate with the one derived from the ROSAT images (Peres et al. 1998), although the presence of a cooling flow is not strongly required by the overall spectrum in our energy band. The table also shows that the absorbing column is weakly constrained (due to our energy cut) but is in good agreement with the Galactic value of $4.2 \times 10^{20} \mathrm{~cm}^{-2}$ (Dickey \& Lockman 1990). We therefore fix $N_{\mathrm{H}}$ at its Galactic value in the analysis below.

\subsection{Temperature Map}

Using Chandra data, it is possible to derive a twodimensional temperature map within $3^{\prime}-4^{\prime}$ of the cluster peak. The Chandra angular resolution is more than sufficient to allow us to ignore any energy-dependent PSF effects and, for example, simply convert an X-ray hardness ratio at each cluster position to temperature. Taking advantage of this simplicity, we also tried to use as much spectral information as possible without dividing the cluster into any regions for full spectral fitting. To do this, we extracted images in five energy (or PI) bands 1.0-1.5-2.0-3.0-5.5-10 $\mathrm{keV}$, smoothed them, and for each $2^{\prime \prime} \times 2^{\prime \prime}$ pixel fitted a spectrum consisting of the flux values in each band properly weighted by their statistical errors. The corresponding background images were created as described in $\S 2$ and

TABLE 1

Overall SpeCtrum Fits

\begin{tabular}{lccccc}
\hline \hline \multicolumn{1}{c}{ Model } & $\begin{array}{c}T_{e} \\
(\mathrm{keV})\end{array}$ & $\begin{array}{c}N_{\mathrm{H}} \\
\left(10^{20} \mathrm{~cm}^{-2}\right)\end{array}$ & Abundance & $\begin{array}{c}\dot{M} \\
\left(h^{-2} M_{\odot} \mathrm{yr}^{-1}\right)\end{array}$ & $\chi^{2} /$ d.o.f \\
\hline Single- $T$ & $8.1 \pm 0.4$ & $3.8 \pm 1.5$ & $0.27 \pm 0.04$ & $\ldots$ & $517.2 / 493$ \\
Cooling flow & $8.8_{-0.9}^{+1.2}$ & $5.9 \pm 2.8$ & $0.28 \pm 0.04$ & $69_{-}^{+70}$ & $515.1 / 492$ \\
\hline
\end{tabular}


subtracted from each image. The background-subtracted images were approximately corrected for the frame transfer smearing effect following the description in $\S 2$ and divided by the vignetting factor relative to the on-axis position (within each energy band, the vignetting factor for different energies was weighted using a $10 \mathrm{keV}$ plasma spectrum). The images were then smoothed by a variable-width Gaussian (same for all bands) whose $\sigma$ varied from $10^{\prime \prime}$ at the cluster peak to $30^{\prime \prime}$ near the edges of the map. Bright point sources were masked prior to smoothing. For each pixel of the map, we fitted a one-temperature plasma model with the absorption column fixed at the Galactic value and iron abundance at the cluster average, multiplying the model by the on-axis values of the telescope effective area (since the images were vignetting-corrected). The instrument spectral response matrix was properly binned for our chosen energy bands.

The resulting temperature map is shown in Figure 3. The useful exposure of our observations is relatively short, so the statistical accuracy is limited. The map shows that the cluster brightness peak is cool and that this cool dense gas is displaced to the SE from the main galaxy G1. There is also a cool filament extending from the peak in the general direction of the second galaxy G2, or along the southern brightness edge. The G2 galaxy itself is not associated with any features in the temperature map.

At larger scales, the map shows that the hottest cluster gas lies immediately outside the NW brightness edge and to the south of the southern edge. In the relatively small region of the cluster covered by our analysis, our temperature map is in general agreement with the coarser ROSAT/Ginga map of Henry \& Briel (1996). Both maps show that the center of the cluster is cool (probably has a cooling flow) and the hot gas lies outside, mostly to the north and west. The maps differ in details; for example, our map indicates an increase of the temperature southeast of the center where

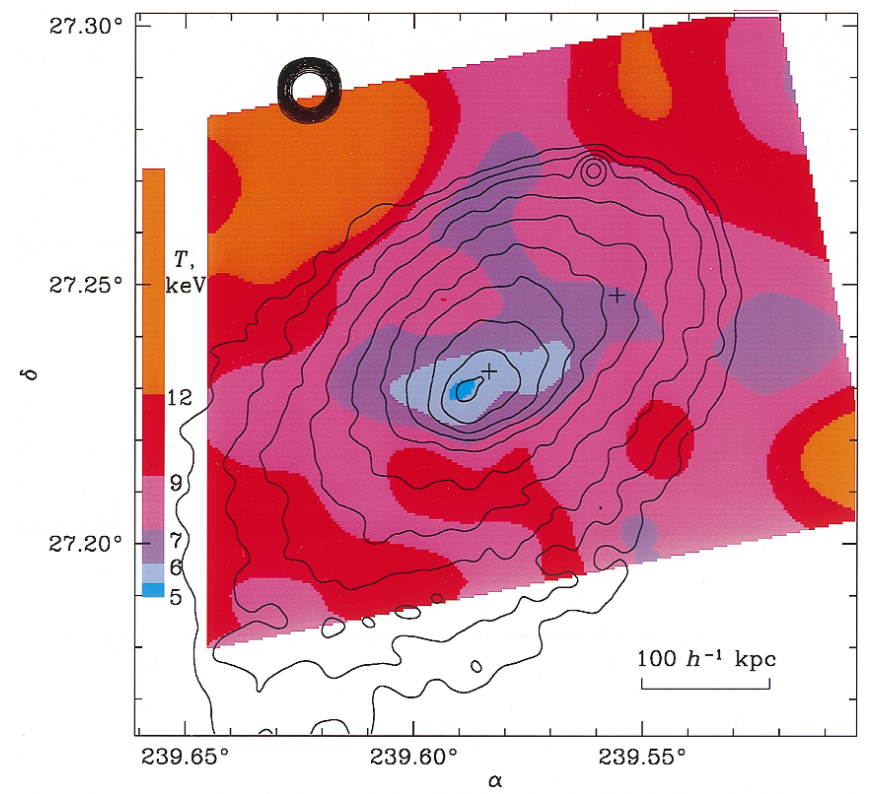

FIG. 3.-Temperature map of the central region of A2142 (color) overlaid on the $0.3-10 \mathrm{keV}$ ACIS brightness contours. The $90 \%$ temperature uncertainties increase from approximately $\pm 0.5 \mathrm{keV}$ at the brightness peak to $\pm 1.5 \mathrm{keV}$ at the outer contour and still greater outside that contour. Crosses denote positions of the two brightest galaxies G1 (center) and $\mathrm{G} 2$ (northwest). the ROSAT map suggests a decrease. An important conclusion from our map is that the brightness edges separate regions of cool and hot gas. These edges are studied in more detail in sections below.

There is also some marginal evidence in Figure 3 for a faint cool filament running across the whole map through the cluster brightness peak and coincident with the chip quadrant boundary. It is within the statistical uncertainties and most probably results from some presently unknown detector effect. This feature does not affect our arguments.

\subsection{Temperature Profiles across the Edges}

To derive the temperature profiles across the edges, we divide the cluster into elliptical sectors as shown in Figure $4 a$, chosen so that the cluster edges lie exactly at the boundaries of certain sectors, and so that the sectors cover the azimuthal angles where the edges are most prominent. Figure $4 b$ shows the best-fit temperature values in each region, for both observations fitted together or separately (for a consistency check). The fitting was performed as described in $\S 3.2$. The temperatures shown in the figure correspond to the iron abundance fixed at the cluster's average and a fixed Galactic absorption; when fitted as a free parameter, the absorption column was consistent with the Galactic value in all regions. For both edges, as we move from the inside of the edge to the outer, less dense region, the temperature increases abruptly and significantly. The profiles also show a decrease of the temperature in the very center of the cluster, which is also seen in the temperature map in Figure 3.

We must note here that our spectral results in the outer, low surface brightness regions of the cluster depend significantly on the background subtraction. To quantify the corresponding uncertainty, we varied the background normalization by $\pm 10 \%$ (synchronously for the two observations), refitted the temperatures in all sectors and added the resulting difference in quadrature to the $90 \%$ statistical uncertainties. While the values for the brighter cluster regions are practically unaffected, for the regions on the outer side of the NW edge, these differences are comparable to the statistical uncertainty. The $10 \%$ estimate is rather arbitrary and appears to overestimate the observed variation of the ACIS quiescent particle background with time. A possible incomplete screening of background flares is another source of uncertainty that is difficult to quantify. Experimenting with different screening criteria shows that it can significantly affect the results. An approximate estimate of this uncertainty is made by comparing separate fits to the two observations (Fig. 4b, dotted crosses); their mutual consistency shows that for the conservative data screening that we used, this uncertainty is probably not greater than the already included error components.

\subsection{Density and Pressure Profiles}

Figure $4 c$ shows $\mathrm{X}$-ray surface brightness profiles across the two edges, derived using narrow elliptical sectors parallel to those used above for the temperatures. The energy band for these profiles is restricted to $0.5-3 \mathrm{keV}$ to minimize the dependence of X-ray emissivity on temperature and to maximize the signal-to-noise ratio. Both profiles clearly show the sharp edges; the radial derivative of the surface brightness is discontinuous on a scale smaller than $5^{\prime \prime}-10^{\prime \prime}$ (or about $5-10 h^{-1} \mathrm{kpc}$, limited mostly by the accuracy with which our regions can be made parallel to the edges). 


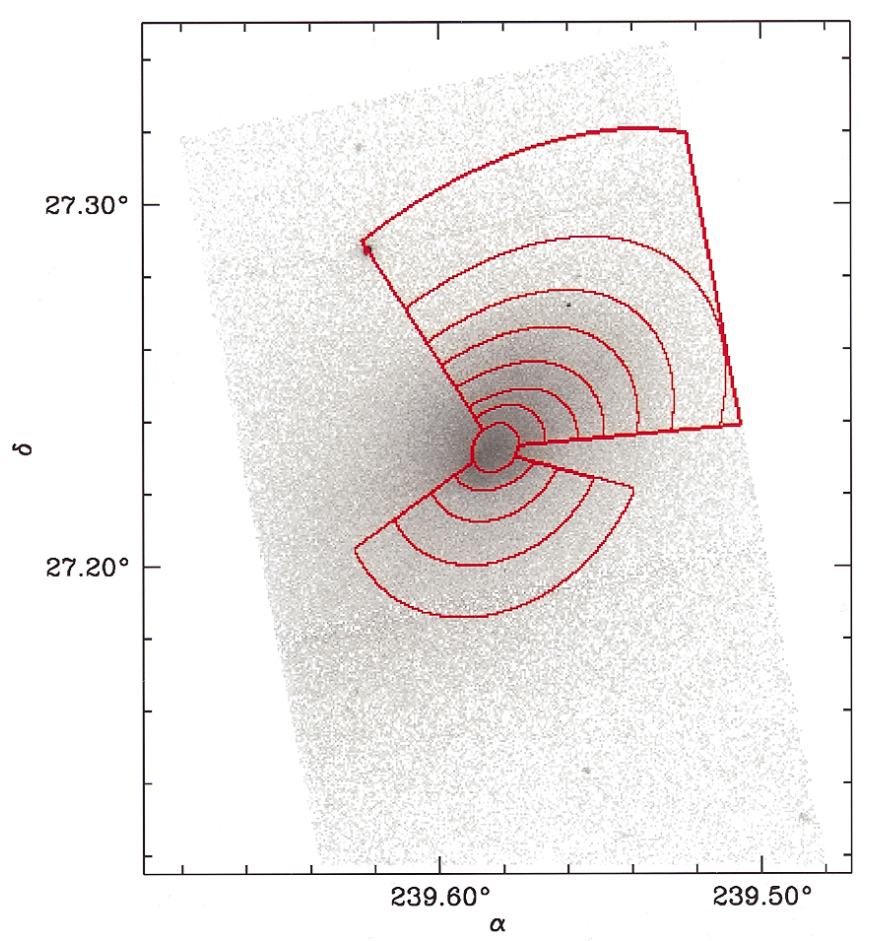

FIG. $4 a$

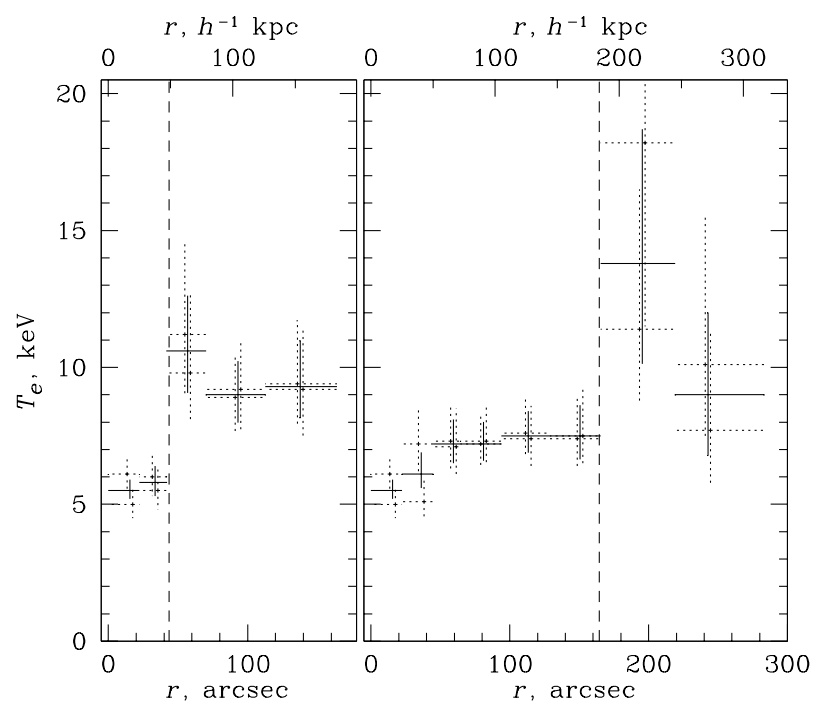

FIG. $4 b$

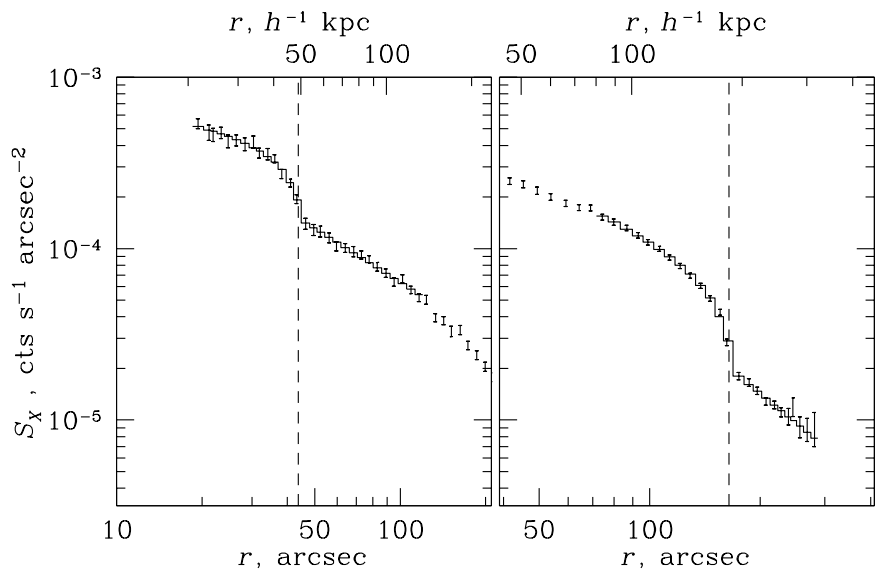

FIG. $4 c$

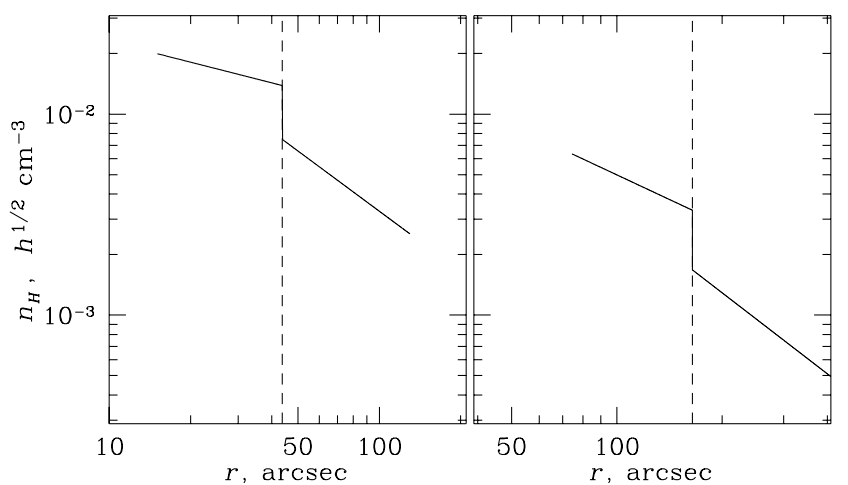

FIG. $4 d$

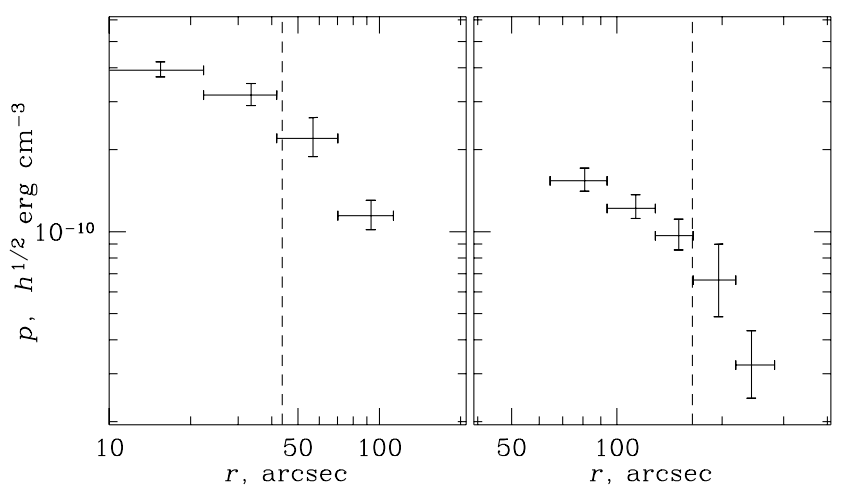

FIG. $4 e$

FIG. 4.- (a) Cluster X-ray image (same as in Fig. 2a); red overlay shows regions centered on the main galaxy G1 and used for derivation of temperature profiles presented in $(b)$. The boundaries are chosen to highlight the brightness edges. Bright point sources are excluded from the regions (not shown for clarity). In $(b)-(e)$, the southern edge is shown in left plot and the northwestern edge is in right plot (the central bin is same for both). In (b), solid crosses show simultaneous temperature fits to both observations, and dashed crosses show separate fits to each observation. Errors are $90 \%$ and, for simultaneous fits, include background uncertainties. The $r$-coordinate for the elliptical sectors corresponds to the emission-weighted distance from the center. Panel (c) shows $\mathrm{X}$-ray brightness profiles across the edges. They are derived using sectors parallel to the elliptical boundaries in $(a)$ but with a finer step (for the southern edge, we also used a somewhat narrower wedge angle for sharpness). Data points are shown as $90 \%$ error bars; the histogram is the best-fit brightness model that corresponds to the gas density model shown in $(d)$. Panel $(e)$ shows pressure profiles obtained from the temperature and density profiles from $(b)$ and $(d)$. Vertical dashed lines show the best-fit positions of the density jumps.

The brightness edges have a very characteristic shape that indicates a discontinuity in the gas density profile. To quantify these discontinuities, we fitted the brightness profiles with a simple radial density model with two power laws separated by a jump. The curvature of the edge surfaces along the line of sight is unknown; therefore, for simplicity, we projected the density model under the assumption of spherical symmetry with the average radius as the single radial coordinate, even though the profiles are derived in elliptical regions. The accuracy of such modeling is sufficient for our purposes. We also restrict the fitting range to the immediate vicinity of the brightness edges (see Fig. 4c) and ignore the gas temperature variations since they are unimportant for the energy band we use. The free parameters are the two power-law slopes and the position and amplitude of the density jump. The best-fit density models 
are shown in Figure $4 d$, and the corresponding brightness profiles are overlaid as histograms on the data points in Figure $4 c$. The best-fit amplitudes of the density jumps are given by factors of $1.85 \pm 0.10$ and $2.0 \pm 0.1$ for the $S$ and NW edges, respectively. As Figure $4 c$ shows, the fits are very good, with respective $\chi^{2}=26.5 / 25$ d.o.f. and 18.9/22 d.o.f. The goodness of fits suggests that the curvature of the edges along the line of sight is indeed fairly close to that in the plane of the sky. To estimate how model-dependent are the derived amplitudes of the jumps, we tried to add a constant density background (positive or negative) as another fitting component representing possible deviations of the profile from the power law at large radii. The resulting changes of the best-fit jump amplitudes were comparable to the above small uncertainties. Thus, our evaluation of the density discontinuities appears robust, barring strong projection effects that can reduce the apparent density jump at the edge.

From the density and temperature distributions in the vicinity of the brightness edges, we can calculate the pressure profiles. Note that even though the measured temperatures correspond to emission-weighted projections along the line of sight, they are reasonably close to the true three-dimensional temperatures at any given radius, because the X-ray brightness declines steeply with radius. Figure $4 e$ shows pressure profiles calculated by multiplying the measured temperature values and the model density values in each region (the density is taken at the emissionweighted radius for each region). Remarkably, while the temperature and density profiles both exhibit clear discontinuities at the edges, the pressure profiles are consistent with no discontinuity within the uncertainties. Thus, the gas is close to local pressure equilibrium at the density edges. It is also noteworthy that the denser gas inside the edges has lower specific entropy, therefore the edges are convectively stable.

\section{DISCUSSION}

Shock fronts would seem the most natural interpretation for the density discontinuities seen in the X-ray image of A2142. Such an interpretation was proposed for a similar brightness edge seen in the ROSAT image of another merging cluster, A3667 (Markevitch et al. 1999), even though the $A S C A$ temperature map did not entirely support this explanation. However, if these edges in A2142 were shocks, they would be accompanied by a temperature change across the edge in the direction opposite to that observed. Indeed, applying the Rankine-Hugoniot shock jump conditions for a factor of $\sim 2$ density jump and taking the postshock temperature to be $\sim 7.5 \mathrm{keV}$ (the inner regions of the NW edge), one would expect to find a $T \simeq 4$ $\mathrm{keV}$ gas in front of the shock (i.e., on the side of the edge away from the cluster center). This is inconsistent with the observed clear increase of the temperature across both edges and the equivalent increase of the specific entropy. This appears to exclude the shock interpretation. An alternative is proposed below.

\subsection{Stripping of Cool Cores by Shocked Gas}

The smooth, comet-like shape and sharpness of the edges alone (especially of the NW edge, see Fig. 2) may hint that we are observing a body of dense gas moving through and being stripped by a less dense surrounding gas. This dense body may be the surviving core of one of the merged sub-
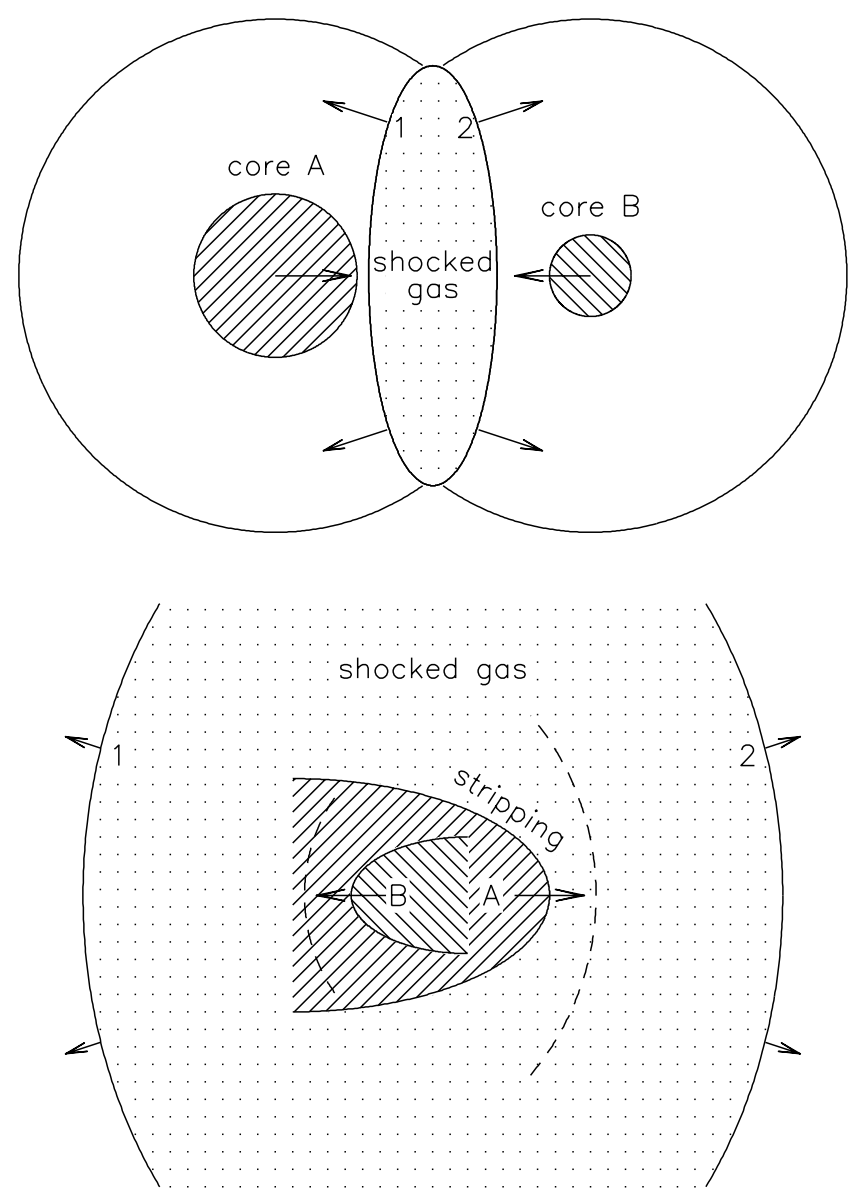

FIG. 5.-Bottom: Model for A2142 proposed in $\S 4.1$ is shown schematically. Top: The preceding stage of the merger is shown. The shaded circles depict dense cores of the two colliding subclusters (of course, in reality, there is a continuous density gradient). Shock fronts 1 and 2 in the central region (top) have propagated to the cluster outskirts (bottom), failing to penetrate the dense cores that continue to move through the shocked gas (core B is likely to be projected onto, or only grazing, core A). The cores may develop additional shock fronts ahead of them, shown by dashed lines. Arrows show velocities of the cores and shocks. Scales and angles are arbitrary.

clusters that has not been penetrated by the merger shocks due to its high initial pressure. The edge observed in the X-ray image could then be the surface where the pressure in the dense core gas is in balance with the thermal plus ram pressure of the surrounding gas; all core gas at higher radii that initially had a lower pressure has been stripped and left behind (possibly creating a tail seen as a general elongation to the SE). A possibility of survival of a dense cluster core in a merger was considered by Fabian \& Daines (1991). The hotter, rarefied gas beyond the NW edge can be the result of earlier shock heating of the outer atmospheres of the two colliding subclusters, as schematically shown in Figure 5. In this scenario, the outer subcluster gas has been stopped by the collision shock, while the dense cores (or, more precisely, regions of the subclusters where the pressure exceeded that of the shocked gas in front of them, which prevented the shock from penetrating them) continued to move ahead through the shocked gas. The southern edge may delineate the remnant of the second core (core B in Fig. 5) that was more dense and compact and still retains a cooling flow. The two cores should have already passed the point of minimum separation and be moving apart at present. It is 
unlikely that the less dense core A could survive a head-on passage of the denser core (in that case we probably would not see the NW edge). This suggests a nonzero impact parameter; for example, the cores could have been separated along the line of sight during the passage, with core B either grazing or being projected onto core A at present.

Although the thermal pressure profiles in Figure $4 e$ do not suggest any abrupt decline across the edges that could be due to a ram pressure component, at the present accuracy they do not strongly exclude it. To estimate what bulk velocity, $v$, is consistent with the data on the NW edge, we can apply the pressure equilibrium condition to the edge surface, $p_{1}=p_{2}+\rho_{2} v^{2}$, where indices 1 and 2 correspond to quantities inside and outside the edge, respectively. The density jump by a factor of 2 and the $90 \%$ lower limit on the temperature in the nearest outer bin, $T_{2}>10 \mathrm{keV}$, corresponds to an average bulk velocity of the gas in that region of $v<900 \mathrm{~km} \mathrm{~s}^{-1}$. This is consistent with subcluster velocities of order $1000 \mathrm{~km} \mathrm{~s}^{-1}$ expected in a merger such as A2142. Note, however, that this is a very rough estimate because, if our interpretation is correct, the gas velocity would be continuous across the edge and there must be a velocity gradient, as well as a compression with a corresponding temperature increase, immediately outside the edge. Also, if the core moves at an angle to the plane of the sky, the maximum velocity may be higher, since one of its components would be tangential to the contact surface that we can see. In addition, as noted above, projection effects can dilute the density jump, producing an excessively tight limit on the ram pressure. A similar estimate for the southern edge is $v<400 \mathrm{~km} \mathrm{~s}^{-1}$, but it is probably even less firm because of the likely projection of core B onto core A.

Depending on the velocities of the cores relative to the surrounding (previously shocked) gas, they may or may not create additional bow shocks at some distance in front of the edges (Fig. 5, dashed lines). The above upper limit on the velocity of core A is lower than the sound velocity in a $T>10 \mathrm{keV}$ gas $\left(v_{s}>1600 \mathrm{~km} \mathrm{~s}^{-1}\right)$ and is therefore consistent with no shock, although it does not exclude it due to the possible projection and orientation effects mentioned above. The available X-ray image and temperature map do not show any obvious corresponding features, but deeper exposures might reveal such shocks.

Comparison of the X-ray and optical images (Fig. 2b) offers an attractive possibility that core $\mathrm{B}$ is centered on galaxy G1 and core A on galaxy G2. However, this scenario has certain problems. Velocity data, while scarce $(\sim 10$ galaxy velocities in the central region; Oegerle et al.), show that $\mathrm{G} 2$ is separated from most other cluster members by a line-of-sight velocity of $\sim 1800 \mathrm{~km} \mathrm{~s}^{-1}$, except for the radio galaxy north of G2 that has a similar velocity. It therefore appears unlikely that G2 can be the center of a relatively big, $T \simeq 7 \mathrm{keV}$ subcluster, unless a deeper spectroscopic study reveals a concentration of nearby galaxies with similar velocity. It is possible that this galaxy is completely unrelated to core $\mathrm{A}$; we recall that it does not display any strong X-ray brightness enhancement. Another problem is a displacement, in the wrong direction, of the cool density peak from the G1 galaxy. If G1 is at the peak of the gravitational potential of the smaller core $B$, one would expect the gas to lag behind the galaxy as the core moves to the south or southeast (as the edge suggests). The observed displacement might be explained if at present core $\mathrm{B}$ is moving mostly along the line of sight on a circular orbit and the central galaxy is already starting its turnaround toward G2, perhaps leaving behind a trail of cool gas seen as a cool filament. The observed southern edge would then be a surface where the relative gas motion is mostly tangential, which is also in better agreement with the low allowed ram pressure.

Below we propose a slightly different scenario for the merger, motivated by comparison of the observed structure with hydrodynamic simulations. It invokes the same physical mechanism for the observed density edges.

\subsection{Late Stage Unequal Mass Merger}

As noted above, our temperature map is substantially in agreement with the coarser map derived by Henry \& Briel (1996) using ROSAT PSPC. The ROSAT map covers greater area than the Chandra data and shows a hot sector extending to large radii in the NW. If this is correct, then a comparison of the X-ray structure with some hydrodynamic simulations (e.g., Roetigger, Loken \& Burns 1997, hereafter RLB97) suggests that A2142 is the result of an unequal merger, viewed at a time at least 1-2 Gyr after the initial core crossing. The late phase is required by the largely smooth and symmetrical structure of the X-ray emission, and the lack of obvious shocks. In the simulations, shock-heated gas at the location of the initial impact of the smaller system can still be seen at late times, similar to the hot sector seen in the Henry \& Briel map far to the NW. Hence, in this model, the low-mass system has impacted from the NW.

The undisrupted cool core which we see in A2142 differs from what is seen in the work of RLB97 and many others. However, these simulations all involved clusters with low core gas densities $\left(n<10^{-3} \mathrm{~cm}^{-3}\right)$. Under these circumstances, the shock runs straight through the core of the main cluster, raising its temperature. In contrast, it appears that the collision shock has failed to penetrate the core of A2142, in which gas densities reach $\sim 10^{-2} \mathrm{~cm}^{-3}$, and has instead propagated around the outside, heating the gas to the north and southwest of the cluster core.

In this model, galaxy G1 is identified with the center of the main cluster (whose core includes the whole elliptical central region of A2142), and there is less difficulty in accepting G2 (which lies essentially along the collision axis, at least in projection) as being the former central galaxy of the smaller subcluster. Having lost its gas halo on entering the cluster from the NW, G2 has already crossed the center of the main cluster twice and is now either returning to the NW, or falling back toward the center for a third time. The latter option derives some support from the fact that the radio galaxy, presumably (from its similar line-of-sight velocity) accompanying $\mathrm{G} 2$, has a narrow-angle radio tail which points to the west, away from the center of the main cluster (Bliton et al. 1998). The idea that G2 has already crossed the cluster core also helps to explain the elongated morphology of the central cooling flow, apparent in Figure 3.

Simulations show that as the subcluster recrosses the cluster core, gas which has been pulled out to the SE should fall in behind it, forming an extended inflowing plume (see, e.g., RLB97, Fig. 8f). This is consistent with the shallow X-ray surface brightness gradient seen to the SE in Figure $2 b$. In the case of $\mathrm{A} 2142$, this gas, flowing in from the SE, will run into the dense cool core surrounding G1 at sub- 
sonic velocity, and this could give rise to the SE density step through a physical mechanism similar to that discussed in the previous section, involving gas shear and stripping at the interface.

In this scenario, the NW edge may be the fossilized remains of the initial subcluster impact that took place here. Shock heating from this impact has raised the entropy of the gas outside the core to the NW. The shock has propagated into the core until the radius where the pressure (or density) in the core matched the pressure driving the shock. Subsequently, the flow of the shocked gas toward the SE has swept away the outer layer of the core where the shock decayed, leaving the high entropy shocked gas in direct contact with the low entropy unshocked core. Once the gas returns to a hydrostatic configuration, this entropy step manifests itself as a jump in temperature and density of the form seen, while the gas pressure would be continuous across the edge. In contrast to the model from the previous section, little relative motion of the gas to either side of the NW edge is expected at this late merger stage, so there is no current stripping. Simulations are required to investigate how long a sharp edge of the kind observed can persist under these conditions; this depends in part on poorly understood factors such as the thermal conductivity of the gas.

\section{SUMMARY}

We have presented the results of a short Chandra observation of the merging cluster A2142, which include a temperature map of its central region and the temperature and density profiles across the two remarkable surface bright- ness edges. The data indicate that these edges cannot be shock fronts - the dense gas inside the edges is cooler than the gas outside. It is likely that the edges delineate the dense subcluster core(s) that survived merger and shock heating of their surrounding, less dense atmospheres. We propose that the edges themselves are surfaces where these cores are presently being ram pressure-stripped by the surrounding hot gas, or fossilized remains of such stripping which took place earlier in the merger. More accurate temperature and pressure profiles for the edge regions would help to determine whether the gas stripping is continuing at present and may also provide information on the gas thermal conductivity. A comprehensive galaxy velocity survey of the cluster, and large-scale temperature maps such as will be available from $X M M$, will help to construct a definitive model for this interesting system.

An accurate quantitative interpretation of the available optical and X-ray data on A2142 requires hydrodynamic simulations of the merger of clusters with realistically dense cores and radiative cooling. We also hope that the results presented here will encourage an improvement in linear resolution of the simulations necessary for modeling the sharp cluster features such as those Chandra can now reveal.

The results presented here are made possible by the successful effort of the entire Chandra team to build, launch, and operate the observatory. Support for this study was provided by NASA contract NAS8-39073 and by Smithsonian Institution. T. J. P., P. E. J. N., and P. M. thank CfA for hospitality during the course of this study.
Allen, S. W., \& Fabian, A. C. 1998, MNRAS, 297, L57

Anders, E., \& Grevesse, N. 1989, Geochim. Cosmochim. Acta, 53, 197

Arnaud, K. A. 1996, in ASP Conf. Ser. 101, Astronomical Data Analysis Software and Systems V, ed. G. Jacoby \& J. Barnes (San Francisco: ASP), 17

Bliton, M., Rizza, E., Burns, J. O., Owen, F. N., \& Ledlow, M. J. 1998, MNRAS, 301, 609

Buote, D. A., \& Tsai, J. C. 1996, ApJ, 458, 27

Dickey, J. M., \& Lockman, F. J. 1990, ARA\&A, 28, 215

Fabian, A. C., \& Daines, S. J. 1991, MNRAS, 252, 17P

Harris, D. E., Bahcall, N. A., \& Strom, R. G. 1977, A\&A, 60, 27

Henry, J. P., \& Briel, U. G. 1996, ApJ, 472, 137

\section{REFERENCES}

Markevitch, M., Forman, W. R., Sarazin, C. L., \& Vikhlinin, A. 1998, ApJ, 503,77

Markevitch, M., Sarazin, C. L., \& Vikhlinin, A. 1999, ApJ, 521, 526

Oegerle, W. R., Hill, J. M., \& Fitchett, M. J. 1995, AJ, 110, 32

Peres, C. B., Fabian, A. C., Edge, A. C., Allen, S. W., Johnstone, R. M., \& White, D. A. 1998, MNRAS, 298, 416

Raymond, J. C., \& Smith, B. W. 1977, ApJS, 35, 419

Roettiger, K., Burns, J. O., \& Stone, J. M. 1999, ApJ, 518, 603

Roettiger, K., Loken, C., \& Burns, J. O. 1997, ApJS, 109, 307 (RLB97)

Schindler, S., \& Müller, E. 1993, A\&A, 272, 137

White, R. E., Day, C. S. R., Hatsukade, I., \& Hughes, J. P. 1994, ApJ, 433, 583 